\title{
Fatigue Crack Growth Life Prediction of Screw Blade in Screw Sand Washing Machine based on Austen Growth Law
}

\author{
Jie Gong ${ }^{1, ~ a}$, Fan Zhang ${ }^{1, b}$, Pu-Wang Li ${ }^{1, c}$, Ji-Hua Li ${ }^{1, d}$, Si-Dong Li ${ }^{1,}$, Zi-Ming \\ Yang ${ }^{1, f_{*}}$
}

${ }^{1}$ Agricultural Product Processing Research Institute at Chinese Academy of Tropical Agricultural Sciences, Chinese Agricultural Ministry Key Laboratory of Tropical Crop Products Processing, Zhanjiang524001, China

a18813611566@163.com, b18675991432@163.com, 'puwangli@163.com, dfoodpaper@163.com, efoodpaper@163.com, yangziming2004@163.com

Keywords: Fatigue crack growth life, Screw blade, Random load with gauss distribution, Linear elastic fracture mechanics, Austen growth law.

Abstract. The purpose of this research is to assess the fatigue crack growth life of the screw blade in the screw sand washing machine. The fatigue crack growth life of the screw blade is assessed on the basis of the random load with the Gauss distribution, $d a / d N-\Delta K$ curve, linear elastic fracture mechanics, and Austen growth law. The results show that the fatigue crack growth life of the screw blade terminates due to fast fracture, and when the initial crack size is $1.00 \mathrm{~mm}$, the fatigue crack growth life of the screw blade is approximately 3.18 years, with a final crack length of $0.048 \mathrm{~m}$.

\section{Introduction}

River sand is an important building material, so the market demand of river sand is extremely huge in the world [1]. With the rapid development of the construction industry, river sand is consumed swiftly, causing that the river sand resource are running out rapidly [1]. To provide the building sand for the construction industry sustainably, marine sand is used widely in the construction industry instead of river sand [1]. However, the chloride ion in marine sand can corrode the steel bar in the concrete, which will result in the reduction of the service life of buildings [2,3]. At present, a number of sand washing technologies are utilized to get rid of the chloride ion in marine sand, and the mechanical sand washing technique is the most popular [4]. Although the cost of the mechanical method is the highest, its production efficiency is also the highest [5].

The sand washing machine is a main component of various mechanical sand washing systems [5]. As the screw sand washing machine has numerous merits (i.e., the long screw, seal design, simple structure, strong processing ability, easy maintenance, etc.), it is widely used in the sea sand desalination field [5]. Particularly, the screw blade is an actuator of washing sea sand in screw sand washing machines, with the result that it will suffer from the sophisticated alternating load [6]. As the service life of the screw blade is closely related to the working life of the screw sand washing machine, the service life of the screw blade is an essential index used for evaluating the performance of the screw sand washing machine. Although Gong et al. (2016) and Fu et al. (2017) predicted the fatigue crack initiation life of the screw blade under the random load with the Gauss distribution and extreme load, the investigations concerning the fatigue crack growth life prediction of the screw blade are rarely studied $[6,7]$.

\section{Generation of Fatigue Load Spectrum}

The stress spectrum is the precondition of the fatigue crack growth life prediction. And also, the practice shows that the fatigue load, in most cases, has some randomness [6]. In fact, Ref. [6] has given the method for generating the fatigue load spectrum on the screw blade. In this study, the 
random load with the Gauss distribution generated in Ref. [6] is used to estimate the fatigue crack growth life of the screw blade. The flow diagram of generating fatigue load spectrum is shown in Fig. 1 [6]. The rainflow cycle counting histogram of the random load with the Gauss distribution is shown in Fig. 2 [6]. Virtually, the rainflow cycle counting histogram after extrapolation shown in Fig. 2 is the fatigue load input utilized for assessing the fatigue crack growth life of the screw blade.

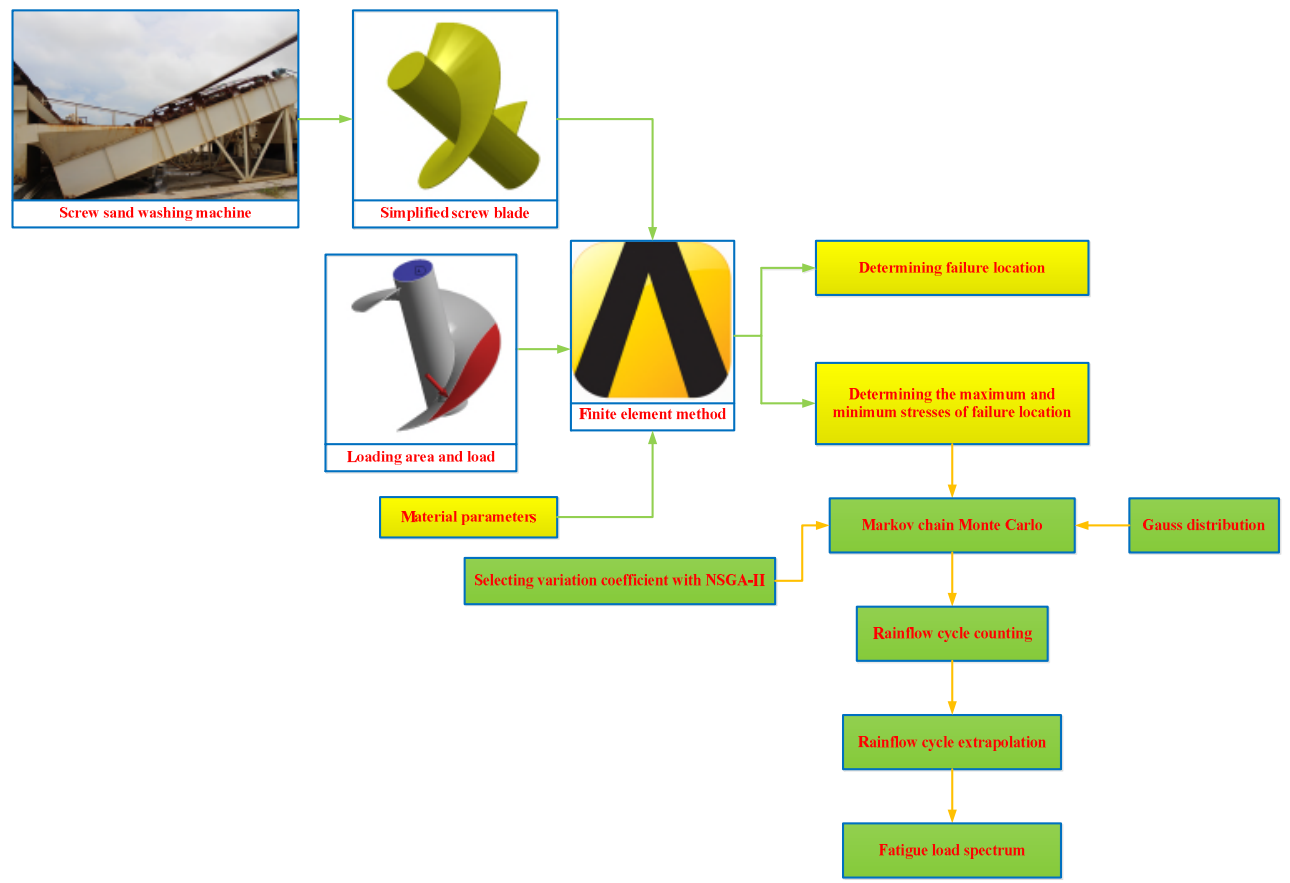

Fig. 1 Flow diagram of generating fatigue load spectrum.
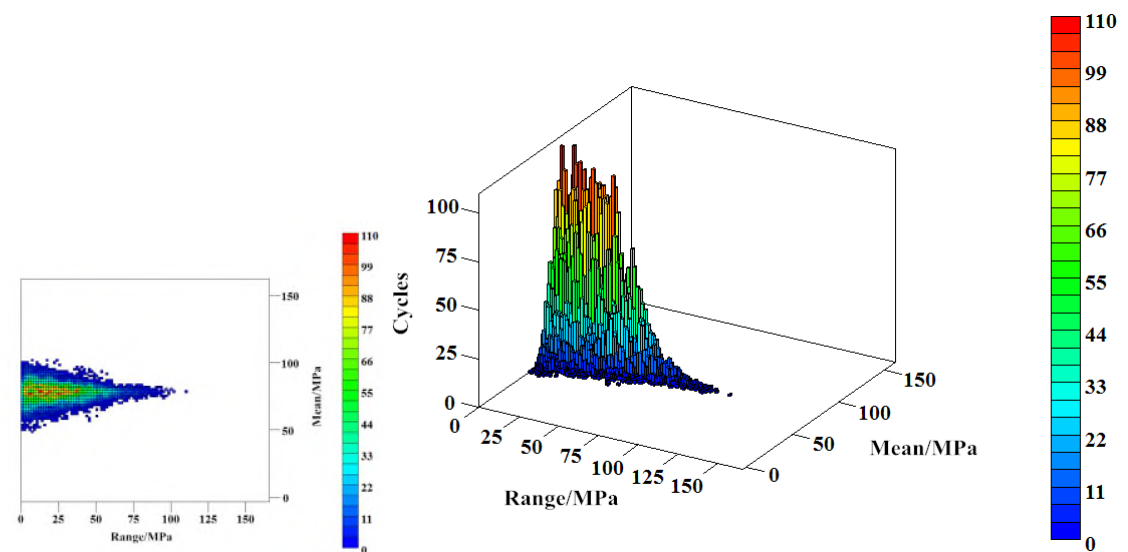

Fig. 2 Rainflow cycle counting histogram of random load with Gauss distribution.

\section{Crack Growth Life of Screw Blade}

The estimation of the crack growth life is based on linear elastic fracture mechanics (LEFM). The Crack Growth module in nCode GlyphWorks is used to estimate the crack growth life of the screw blade. The formula of the fatigue crack growth rate is directly related to the estimation accuracy of the fatigue crack growth life. The best known crack growth law is the Paris Law [8, 9]:

$$
d a / d N=C(\Delta K)^{n}
$$

Where $d a / d N$ is the crack growth rate in meters per cycle, $\Delta K$ is the stress intensity factor range in newton-meters ${ }^{-3 / 2}, C$ is the Paris law coefficient in meters, and $n$ is the Paris law exponent.

The relationship between $\Delta K$, stress and crack size is [10, 11] 


$$
\Delta K=Y \Delta \sigma \sqrt{\pi a}
$$

where $\Delta \sigma$ is the stress range of the cycle in megapascals, $a$ is the current crack size in meters, and $Y$ is a factor that depends on the geometry of the specimen, the location of the crack, and the loading configuration. The factor $Y$ can be expressed by $[10,11]$

$$
Y=C_{1}+C_{2}(a / W)+C_{3}(a / W)^{2}+C_{4}(a / W)^{3}+C_{5}(a / W)^{4}
$$

Where $C_{1}$ to $C_{5}$ are the correction coefficients and $W$ is the geometrical width in meters.

Compared with the crack growth life calculated by other growth models (i.e., Interpolation Forman, NASGRO, FASTRAN, and AFGROW), the crack growth life calculated by Austen growth model is the most conservative [12], so this study focuses on the Austen growth law for calculating the crack growth life of the screw blade. The Austen growth model is expressed by [12]

$$
d a / d N=C\left(\Delta K_{e f f}\right)^{n}
$$

where $\Delta K_{\text {eff }}=\Delta K_{\max e f f}-\Delta K_{\min e f f}, \quad K_{\max e f f}=K_{\max }+K_{S F}, \quad K_{\min e f f}=-\max \left(K_{\min }, K_{C L}\right), \quad K_{S F}$ is the modification for static fracture in newton-meters ${ }^{-3 / 2}$, and $K_{C L}$ is the stress intensity at the crack closure in newton-meters ${ }^{-3 / 2}$. Furthermore, Austen modelled the onset of fast fracture using the following expression [12]:

$$
K_{S F}=K_{\max } /\left(K_{1 C}-K_{\max }\right)
$$

Where $K_{1 C}$ is the plane strain fracture toughness in newton-meters ${ }^{-3 / 2}$.

Austen also takes account of the threshold and short cracks by applying a crack closure stress $K_{1 C}$ expressed as [12]

$$
K_{C L}=K_{\max }-K_{\max } \sqrt{\left(a+I_{0}\right) / a}+\Delta K_{t h} /(1-R)
$$

where $I_{0}$ is the smallest crack size in meters and $R$ is the stress ratio.

The smallest crack size $I_{0}$ is given by [13]

$$
I_{0}=1 / \pi\left(\Delta K_{t h} / \Delta \sigma_{0}\right)^{2}
$$

where $\Delta \sigma_{0}$ is the un-notched fatigue strength in megapascals and $\Delta K_{\text {th }}$ is the fatigue crack growth threshold in newton-meters ${ }^{-3 / 2}$.

The fatigue crack growth threshold $\Delta K_{\text {th }}$ denotes fracture strength of material subjected to cyclic loading and is defined as the lower limit value of $\Delta K$ to induce fatigue crack growth. The fatigue crack growth threshold $\Delta K_{t h}$ can be described by the equation [13]

$$
\Delta K_{\text {th }}=D_{0}-\left(D_{0}-D_{1}\right) R / R_{\text {crit }}
$$

where $D_{0}$ is the $\Delta K$ at $R=0$ in newton-meters ${ }^{-3 / 2}, \quad D_{1}$ is the $\Delta K$ as $R->1$ in newton-meters $^{-3 / 2}$, and $R_{\text {crit }}$ is the value of $R$ above which $\Delta K_{\text {th }}$ is constant and equal to $D_{1}$.

Let the initial crack size be $1.00 \mathrm{~mm}$, which is a value chosen on the basis of practical considerations [13]. According to actual working conditions, the center cracked square plate in tension is adopted to describe the crack geometry of the screw blade. Related material parameters are as follows:

$$
\begin{aligned}
& K_{1 C}=42.5 \mathrm{~N} \cdot \mathrm{m}^{-3 / 2}, C=6.81 \times 10^{-7} \mathrm{~m}, n=2.25, \\
& D_{0}=4.72, D_{1}=2.81, R_{\text {crit }}=1, \Delta \sigma_{0}=220 \mathrm{MPa}
\end{aligned}
$$

The $d a / d N-\Delta K$ curve of the screw blade is shown in Fig. 3.

The cycle-by-cycle algorithm, which is technically the correct way to calculate crack growth, is 
used to calculate the crack extension resulting from each cycle. This algorithm includes recalculating the crack geometry's stress intensity factor for every cycle. In addition, crack retardation is taken into consideration in the calculation of crack growth [14, 15].

The fatigue crack growth life of the screw blade is assessed based on the random load with the Gauss distribution, $d a / d N-\Delta K$ curve, linear elastic fracture mechanics, and Austen growth law. The crack growth curve of the screw blade is shown in Fig. 4. It can be clearly seen in Fig. 4 that the crack growth analysis of the screw blade terminates due to fast fracture (i.e., static fracture occurs as a result of exceeding the material's fracture toughness.), with a final crack length of 0.048 $\mathrm{m}$. It takes about $2.51 \times 10^{5}$ cycles, which is equal to 3.18 years when the working time of the screw wash-sand machine is 18 hours per day. In other words, if the initial crack of the screw blade is detected, then its residual life is about 3.18 years. In actual engineering, the crack growth life is only a small part of the fatigue life of the screw blade in the screw wash-sand machine.

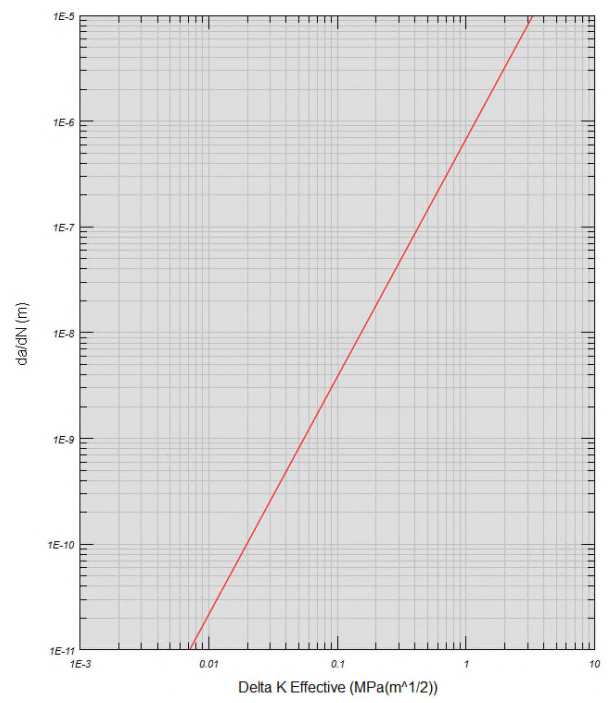

Fig. $3 d a / d N-\Delta K$ curve.

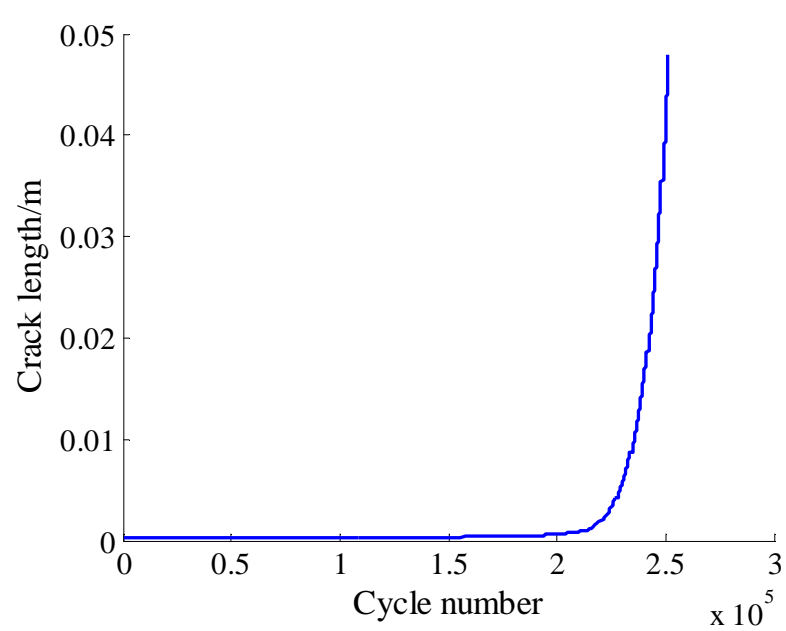

Fig. 4 Crack growth curve.

\section{Conclusions}

(1) The fatigue crack growth life of the screw blade terminates due to fast fracture (i.e., static fracture occurs as a result of exceeding the material's fracture toughness.).

(2) When the initial crack size is $1.00 \mathrm{~mm}$, the fatigue crack growth life of the screw blade is approximately 3.18 years, with a final crack length of $0.048 \mathrm{~m}$.

(3) The study on the fatigue crack growth life prediction of the screw blade could be beneficial to accurately control the fatigue strength of the screw blade on the basis of actual engineering 
requirements. Also, it could provide some theoretical guidance for determining the reasonable maintenance period of the screw sand washing machine.

\section{Acknowledgements}

The authors gratefully acknowledge the Financial Support by the Foundation of Science and Technology Competitive Allocation of Zhanjiang (Project no. 2014A02010), the Funds for Innovation Introduced and Integration Project of Hainan Province (Project no. KJHZ2014-10), and the Fundamental Research Funds for Rubber Research Institute, CATAS (Project no.1630022013019).

\section{References}

[1] Z. M. Yang, S. D. Li, Y. F. Fu, M. Z. Lv, F. Zhang, Z. Hu, P. Li, Research on new desalination method of sea sand and its properties, Guangdong Chem. Ind. 43(18) (2016) 54-56.

[2] W. Liu, H. Z. Cui, Z. J. Dong, F. Xing, H. C. Zhang, Y. Lo. Tommy, Carbonation of concrete made with dredged marine sand and its effect on chloride binding, Construct. Build. Mater. 120 (2016) 1-9.

[3] Y. L. Li, X. L. Zhao, R. K. Raman Singh, S. Al-Saadi, Experimental study on seawater and sea sand concrete filled GFRP and stainless steel tubular stub columns, Thin-Walled Struct. 106 (2016) 390-406.

[4] Y. F. Fu, J. Gong, Z. M. Yang, P. W. Li, S. D. Li, M. Z. Lv, Reliability analysis of mechanical sand washing system, International Conference on Advances in Energy, Environment and Chemical Engineering. Paris: Atlantis Press, 2015, 533-536.

[5] Y. F. Fu, J. Gong, Z. Peng, J. H. Li, S. D. Li, P. W. Li, Z. M. Yang, Optimization design for screw wash-sand machine based on fruit fly optimization algorithm, J. Appl. Sci. Eng. 19(2) (2016) 149-161.

[6] J. Gong, Y. F. Fu, W. Xia, J. H. Li, F. Zhang, Fatigue Life prediction of screw blade in screw sand washing machine under random load with gauss distribution, Am. J. Eng. Appl. Sci. 9(4) (2016) 1198-1212.

[7] Y. F. Fu, J. Gong, P. W. Li, Z. M. Yang, Fatigue life assessment of screw blades in screw sand washing machine under extreme load, Am. J. Mech. Eng. 5(1) (2017) 1-7.

[8] P. C. Paris, P. G. Gomez, W. E. Anderson, A rational analytic theory of fatigue, The Trend Eng. 13 (1961) 9-14.

[9] P. C. Paris, F. Erdogan, A critical analysis of crack propagation laws, J. Basic Eng. 85(4) (1963) 528-534.

[10]N. Recho: Fracture mechanics and crack growth (Wiley, London 2012).

[11]Joshua Pelleg: Mechanical properties of materials (Springer, New York 2012).

[12] S. M. Beden, S. Abdullah, A. K. Ariffin, N. A. Al-Asady, Fatigue crack growth simulation of aluminium alloy under spectrum loadings, Mater. Des. 31(7) (2010) 3449-3456.

[13]J. J. Xiong, R. A. Shenoi: Fatigue and fracture reliability engineering (Springer, New York 2011).

[14]C. Makabe, A. Yamauchi, A. Purnowidodo, T. Miyazaki, Effects of crack opening behavior on crack growth rate after overload, J. Solid Mech. Mater. Eng. 2(2) (2008) 243-253.

[15] S. Daneshpour, J. Dyck, V. Ventzke, N. Huber, Crack retardation mechanism due to overload 
in base material and laser welds of Al alloys, Int. J. Fatigue, 42 (2012) 95-10. 